\title{
Specific knockdown of uPA/uPAR attenuates invasion in glioblastoma cells and xenografts by inhibition of cleavage and trafficking of Notch -1 receptor
}

Hari Raghu' ${ }^{1}$, Christopher S Gondi ${ }^{1}$, Dzung H Dinh ${ }^{2}$, Meena Gujrati ${ }^{3}$ and Jasti S Rao ${ }^{1,2^{*}}$

\begin{abstract}
Background: UPA/UPAR is a multifunctional system that is over expressed in many cancers and plays a critical role in glioblastoma (GBM) invasion. Previous studies from our lab have also shown that uPA/UPAR down regulation inhibits cancer cell invasion in SNB 19 GBM cells.

Methods: As Notch 1 is known to be over expressed and promotes invasion in glioblastoma, we therefore tested our hypothesis of whether down regulation of UPA/UPAR, singly or in tandem, attenuates GBM invasion via Notch 1 receptor. Targeted down regulation of UPA/UPAR, either singly or simultaneously, inhibited the anchorage independent growth of U251MG and GBM xenograft cell lines 4910 and 5310 as assessed by soft agar colony formation assay. Expression of all four Notch receptors was confirmed in GBM tissue array analysis by immunohistochemistry.
\end{abstract}

Results: Down regulation of UPA/UPAR, either singly or simultaneously, in U251 MG and tumor xenografts inhibited the cleavage of the Notch receptor between the Gly 1743 and Val 1744 positions, thereby suggesting inhibition of activated cytosolic fragment-related Notch gene transcription. Morphological analysis confirmed inhibition of NICD when U251 MG cells were treated with PUPA, pUPAR or pU2. UPA/UPAR down regulation inhibited Notch 1 mRNA in all three examined cell lines. UPA/UPAR shRNA down regulated nuclear activation of NF- $\kappa$ B subunits and phosphorylation of AKT/mTOR pathway in U251 MG and GBM xenografts. puPA down regulated NICD and HES induced phosphorylation of AKT/ERK and NF- $\kappa$ B. Down regulation of Notch 1 using siRNA inhibited UPA activity as shown by fibrinogen zymography. It also decreased UPA expression levels as shown by western blotting. Exogenous addition of UPA activated Notch 1 in uPAR antisense U251 MG cells and also in UPAR antisense cells transfected with siRNA against Delta and Jagged. The Notch 1 receptor co-localized with LAMP-1, a marker for lysosomes in UPA, UPAR and U2, down regulated U251 MG cells which probably indicates inhibition of Notch 1 receptor trafficking in GBM cells. Notch 1 expression was significantly inhibited in puPA- and pU2-treated pre-established intracranial tumors in mice.

Conclusions: Overall our results show that down regulation of UPA/UPAR, either singly or simultaneously, could be an effective approach to attenuate Notch 1 receptor cleavage, signaling and endosomal trafficking in U251MG cells and xenografts, and ultimately inhibiting GBM invasion.

Keywords: UPA/UPAR, invasion, glioblastoma, Notch 1 receptor

\footnotetext{
* Correspondence: jsrao@uic.edu

'Department of Cancer Biology and Pharmacology, University of Illinois

College of Medicine, One Illini Drive, Peoria, IL 61605, USA

Full list of author information is available at the end of the article
} 


\section{Background}

In spite of the recent development of targeted therapies (e.g., Temozolomide and avastin), glioblastoma (GBM) still continues to be a medical challenge. Identification of novel targets such as UPA and UPAR that are over expressed in glioblastoma and understanding the different signaling pathways they regulate will greatly enhance the clinical treatment and outcome for patients affected with glioblastoma.

The uPA/uPAR system is a multifunctional system which is involved in several cellular processes like migration, angiogenesis and invasion. Studies by others and us have conclusively shown that the UPA/uPAR system significantly correlates to tumor aggressiveness and poor outcome. Our studies have also shown that shRNA constructs directed against uPA/uPAR, either singly or in combination, have a significant inhibitory effect on the migration, invasion and angiogenesis of GBM cells and xenografts.

Notch signaling is a highly conserved pathway playing an important role during embryo development and adulthood. In mammals, it consists of four receptors, namely Notch 1, Notch 2, Notch 3 and Notch 4 [1,2]. The Notch receptor is a hetero-oligomer and a singlepass transmembrane receptor. The transmembrane monomer is bound through non-covalent interactions to a fully extracellular monomer. Notch is involved in cell proliferation, apoptosis, differentiation, survival and stabilization of arterial endothelial fate, angiogenesis and many other functions $[3,4]$. Notch signaling is deregulated in many cancers including glioblastoma [5]. The Notch receptor contains the extracellular peptide which contains EGF repeats that non-covalently associate with the transmembrane peptide and binds the Notch ligands (e.g. Delta and Jagged). Upon ligand binding, the Notch receptor is cleaved and sensitized by ADAM metalloproteases and gamma secretase. This leads to cleavage of NICD (Notch intracellular domain) to the nucleus, and the NICD forms a complex with CBF-1 and the transcriptional co-activator MAML for transcription of Notch genes [6]. Previous studies have shown that inhibition of Notch signaling by pharmacological or genetic means leads to cell cycle arrest and suppression of cell growth [7-9].

We have shown that UPA and UPAR downregulation inhibits invasion in SNB19 glioma cells by decreasing phosphorylation of the Ras-activated FAK, p38MAPK, JNK and ERK1/2, as well as the MEK-activated phosphatidylinositol 3-kinase, AKT and mTOR pathway, indicative of a feedback signaling mechanism of the uPAR/uPA system [10]. Moreover, studies have shown that Notch 1 signaling is known to cross talk with ERK, NF- $\kappa \mathrm{B}$ and with the PI3-K/AKT/mTOR pathway [1]. Notch has multiple roles in invasion and angiogenesis of many human cancers but the mechanisms are not understood $[11,12]$. In this study, we have shown that down regulation of $\mathrm{UPA}$ and $\mathrm{UPAR}$ inhibits invasion of U251 MG cells and xenograft glioma cells by inhibition of Notch- related gene transcription, signaling mechanism and targeting Notch 1 to the lysosomal pathway. We have also shown that UPA/uPAR down regulation inhibits Notch 1 expression in pre-established tumors in nude mice. Overall, our results suggest that down regulation of $\mathrm{uPA} / \mathrm{uPAR}$, either singly or in combination, results in the inhibition of glioma cell invasion via inhibition of Notch 1 receptor cleavage, signaling and endosomal trafficking of the Notch 1 receptor.

\section{Methods}

\section{Cells and Reagents}

U251 MG cells were obtained from American Type Culture Collection, (Manassas, VA), and glioma xenograft cell lines (5310 \& 4910) were kindly provided by Dr. David James (University of California, San Francisco, San Francisco, CA). All cell lines were cultured as described previously [10]. Antibodies to Notch 1, 2, 3 and 4 were obtained from Santa Cruz Biotechnology (Santa Cruz, CA). Antibodies to cleaved Notch between Gly 1743 and Val 1744 were obtained from Cell Signaling (Beverly MA). Antibodies to extracellular domain of Notch were obtained from Abcam (Cambridge, UK). Antibodies to Delta and Jagged were obtained from Santa Cruz Biotechnology, (Santa Cruz, CA). Antibodies to $\mathrm{RBPJ} \kappa$, Lamin $\mathrm{B}$, alpha tubulin, pAKT, NF- $\kappa \mathrm{B}$ p 65, MEK, pERK, pmTOR Ser 2448, were obtained from Cell Signaling (Beverly MA). Antibodies to uPA and uPAR were purchased from R\&D Systems (Minneapolis, MN). Notch 1 siRNA and antibodies to GAPDH and LAMP-1 were purchased from SCBT (Santa Cruz, CA).

\section{UPA and UPAR shRNA constructs}

shRNA sequences targeting UPAR and UPA were constructed according to our previous publication [13].

\section{Transfection with shRNA constructs}

$1.5 \times 10^{5}$ cells were plated in $100 \mathrm{~mm}$ petri dishes for each transfection experiment. The cells were transfected in serum-free L-15 media using $10 \mu \mathrm{g}$ of Fugene reagent (Roche, USA) according to the manufacturer's instructions. The following constructs were used for transfection: puPA, puPAR, pU2 (both uPA and uPAR) and $\mathrm{pSV}$ (scrambled vector). No plasmid was introduced in the control plates. After 12 hrs of transfection, the serum-free media was replaced with serum-containing media and the cells were left in the incubator at $37^{\circ} \mathrm{C}$ for 48 hrs. The media was then replaced with serumfree media, and conditioned media was collected $12 \mathrm{hrs}$ later. Cells were harvested for isolation of total RNA 
and/or total cell lysates. Conditioned media was used for fibrinogen plasminogen zymography.

\section{Fibrinogen plasminogen zymography}

We used fibrin zymography to determine the activity of the plasminogen activators as previously described [14]. The samples were subjected to SDS-PAGE with 10\% gels that contained fibrinogen and plasminogen. Following electrophoresis, the gels were washed twice with $2.5 \%(\mathrm{v} / \mathrm{v})$ Triton X-100 for $30 \mathrm{~min}$ each time to remove SDS. Finally, the gels were incubated with $0.1 \mathrm{M}$ glycine buffer ( $\mathrm{pH} 7.5$ ) at $37^{\circ} \mathrm{C}$ overnight, stained with amido black, and then destained. The final gel had a uniform background except in regions to which uPA had migrated and cleaved its substrate.

\section{Western blotting}

U251 MG, 5310 and 4910 cells were left untreated or transfected with puPA, puPAR or pU2. Cells were collected and whole cell lysates were prepared by lysing cells in RIPA lysis buffer containing a protease inhibitor cocktail (Sigma, St. Louis, MO). Equal amounts of protein fractions, immunoprecipitates or lysates were resolved by SDS-PAGE and transferred to a polyvinylidene difluoride membrane. Proteins were detected with appropriate primary antibodies followed by HRP-conjugated secondary antibodies. Comparable loading of proteins was verified by reprobing the blots with an antibody specific for the housekeeping gene product, GAPDH.

\section{Reverse transcriptase polymerase chain reaction}

Total RNA was isolated from U251MG, 4910 and 5310 cells using the TRIzol reagent as per the standard protocol. Total RNA was treated with DNAse I (Invitrogen, Carlsbad, CA) to remove contaminating genomic DNA. PCR analysis was done using the one-step reverse transcription-PCR kit (Invitrogen, Carlsbad, CA). Glyceraldehyde 3-phosphate dehydrogenase (GAPDH) was used as an internal control. The following primers were used:

Notch 1 Forward 5'-CAGCTTGCACAACCAGACAGAC-3'

Notch 1 Reverse 5'-ACGGAGTACGGCCCATGTT-3'

Notch 2 Forward AACTGTCAGACCCTGGTGAAC

Notch 2 Reverse CGACAAGTGTAGCCTCCAATC

Notch 3 Forward TGACCGTACTGGCGAGACT

Notch 3 reverse CCGCTTGGCTGCATCAG

Notch 4 Forward AGTCCAGGCCTTGCCAGAACG

Notch 4 Reverse GTAGAAGGCATTGGCCAGAGAG

GAPDH:5'-CGGAGTCAACGGATTTGGTCGTAT3'sense

5'-AGCCTTCTCCATGGTGGTGAAGAC-3' antisense

The PCR conditions were as follows: $95^{\circ} \mathrm{C}$ for $5 \mathrm{~min}$., followed by 30 cycles of $95^{\circ} \mathrm{C}$ for $1 \mathrm{~min}$., and annealing temperature set according to the AT and GC content of the primers.

\section{NF- $\kappa$ B family transcription factor assay}

Nuclear extracts from untreated U251 MG, 5310 and 4910 cells transfected with pSV, puPA, puPAR or pU2 were assayed for activation of NF- $\kappa \mathrm{B}$ transcription factor by TransAM NF- $\kappa$ B family activation kit (Active Motif, Carlsbad, CA) according to the manufacturer's instructions.

\section{Immunofluorescence assay}

U251 MG, 4910 and 5310 cells were either transfected with pSV, puPA, puPAR or pU2 or left untransfected. The cells were then fixed, permeabilized, stained with primary antibodies against Notch 1 and LAMP-1, washed, incubated with Alexa fluor tagged secondary antibodies, washed with PBS and mounted in an antifading reagent containing DAPI. The cells were then visualized using a confocal microscope using Olympus Fluoview software.

\section{UPA/UPAR quantification by ELISA}

Conditioned medium from mock and pSV, puPA, puPAR, or pU2-transfected U251 MG, 5310 and 4910 cells were subjected to ELISA for UPA and UPAR (R\&D Systems, Minneapolis, MN) according to the manufacturer's instructions.

\section{Soft colony agar assay}

Anchorage independent growth was determined by assaying colony formation in soft agar [15]. Briefly, U251 MG, 5310 and 4910 cells which were either untreated or transfected with pSV, puPA, puPAR or pU2 were suspended in DMEM or RPMI containing $10 \%$ FBS and $0.33 \%$ sea plaque low-melting temp agarose. $2 \mathrm{ml}$ containing $2 \times 10^{4}$ cells were plated on a 35 $\mathrm{mm}$ dish over a $3 \mathrm{ml}$ layer of solidified DMEM/RPMI $10 \%$ FBS and $0.6 \%$ agarose. The cells were fed by adding $200 \mu \mathrm{l}$ of RPMI/10\% FBS or with DMEM/10\% FBS. The colonies were photographed at 10x and 20x magnification after 4 weeks.

\section{Matrigel invasion assay}

Invasion of U251MG and 4910 cells was determined by a Matrigel invasion assay as described previously [16]. Briefly, U251MG, 4910 cells which were either left untreated, treated with DAPT (N-[N-(3,5-Difluorophenacetyl)-L-alanyl]-S-phenylglycine t-butyl ester ( NICD inhibitor) for $1 \mathrm{~h}$ at $37^{\circ} \mathrm{C}$ or transfected with pSV, puPA, puPAR or pU2 were detached, washed twice in PBS, suspended in serum-free medium and seeded in the upper chamber of a Transwell insert (8- $\mu \mathrm{m}$ pores) coated with matrigel $\left(1 \mathrm{mg} \mathrm{ml}{ }^{-1}\right)$ (Collaborative 
Research Inc., Boston, MA). The lower chamber was filled with $700 \mu \mathrm{l}$ of complete medium. After $18 \mathrm{hrs}$ of incubation period, the non-migrated cells in the upper chamber were gently scraped away, and invaded cells present on the lower surface of the insert were stained with Hema-3 (Fisher Diagnostics, Middletown, VA). Photographs of the invaded cells were taken with a light microscope (Olympus IX-71, Minneapolis, MN).

\section{Co-localization of Notch 1 with LAMP-1}

Semi-confluent U251 MG cells grown in 8-well chamber slides were left untreated or transfected with pSV,puPA, puPAR and pU2. After $48 \mathrm{hrs}$, the cells were washed, fixed with $2 \%$ paraformaldehyde, permeabilized in $0.5 \%$ Triton X-100, and stained with primary antibodies against Notch-1 and LAMP-1. The cells were then washed and further incubated with appropriate secondary Alexa fluor antibodies, and examined under an Olympus Fluoview microscope.

\section{Animal experiments}

U251 MG cells $\left(2 \times 10^{6}\right)$ were injected into the brains of nude mice using a stereotactic frame. After 8-10 days the mice were treated with pSV, puPAR, puPA and $\mathrm{pU}$. The in vivo intracranial delivery of the vectors was performed using Alzet mini-osmotic pumps (Direct Corp, Cupertion, CA) at the rate of $0.25 \mu \mathrm{l} / \mathrm{h}$ [mock (PBS), $150 \mu \mathrm{g}$ of Vector DNA, $150 \mu \mathrm{g}$ of puPA, $150 \mu \mathrm{g}$ of puPAR and $150 \mu \mathrm{g}$ of pU2]. All experiments were performed in compliance with institutional guidelines established by the University Of Illinois College Of Medicine at Peoria. After five weeks or when the control mice started showing symptoms, mice were euthanized by cardiac perfusion with formaldehyde. The brains were then removed and paraffin embedded following standard protocols. Sections were prepared with H\&E. In each case sections were blindly reviewed and scored semi-quantitatively for tumor size. Sections were observed and immunohistochemical analysis for Notch 1 was done on paraffin-embedded brain tumor sections of mice treated with pSV, puPA and pU2 with specific antibody for Notch 1 .

\section{Results and Discussion}

Notch receptors are expressed in human glioblastoma tissue microarray and in glioblastoma cells and glioma xenograft cell lines

To determine if Notch is expressed in glioblastoma tissues and cells, we wanted to check for Notch receptor expression by GBM human tissue array, western blot and by RT-PCR in U251 MG, 4910 and 5310 cells. Consistent with previous reports, Notch 1, Notch 2, Notch 3 and Notch 4 expression by immunohistochemistry was observed in the GBM tissue array (Figure 1A, panels a, c, e, and $g$ ). Notch receptors 1 to 4 were minimally expressed in normal brain tissue. (Figure 1A, panels b, d, $f, h)$ Further confirmation of our GBM tissue array results was accomplished with immunofluorescent analysis (data not shown). Lysates from all the three cell lines showed expression of Notch 1, 2, 3, and 4 (Figure 1B) and results of mRNA analysis by RT-PCR also showed gene expression of all the four receptors (Figure $1 \mathrm{C}$ ).

\section{shRNA-mediated UPA/uPAR downregulation inhibits anchorage independent growth of U251 MG, 5310 and 4910 cells}

U251 MG, 5310, 4910 cells were left untransfected or transfected with puPA, puPAR and pU2 were analyzed by a colony formation assay in soft agar (Figure 1D). puPA-, puPAR- and pU2-transfected U251 MG (Figure 1D, panel a to e), 5310 (Figure 1D, panel $f$ to $j$ ) and 4910 (Figure 1D, panel $\mathrm{k}$ to o) cells showed significant decreases in colony number $(* * \mathrm{p}<0.0001)$. Quantitative analysis of the number of anchorage independent colonies in soft agar after shRNA transfection in U251 MG, 5310 and 4910 cells is shown in Figure 1E. The colony size was also decreased in the shRNA-transfected cells in comparison to the controls. A complete recovery in both colony number and size was obtained when exogenous uPA and ruPAR was added (data not shown) indicating a specific role for UPA and UPAR in anchorage-independent growth of U251, 5310 and 4910 cells. To further confirm our results, we performed a matrigel invasion assay in U251MG and 4910 cells left untransfected or transfected with SV, puPA, puPAR and pU2 and with DAPT (an NICD inhibitor). Results showed that invasion was inhibited in puPA, puPAR, pU2 and DAPT-treated conditions compared with controls, suggesting that inhibition of invasion by shRNA constructs against $\mathrm{UPA}, \mathrm{UPAR}$ and $\mathrm{U} 2$ is mediated by Notch-1 receptor (Figure $1 \mathrm{~F}$ ).

puPA, puPAR and pU2 inhibit cleavage of Notch receptor in U251MG and xenograft cell lines

Notch receptor is cleaved by four enzymes and each cleaved product is significant to its function. However, cleavage of Notch 1 by gamma secretase is very critical for the function of the Notch receptor. Cleavage of Notch 1 by $\gamma$ secreatase results in the release of NICD (Notch intracellular domain), and release of NICD to the nucleus is critical for Notch gene expression. In all of the three cell lines examined, we observed that puPA, puPAR and pU2 inhibited the cleavage of the Notch receptor as indicated by the absence of a cleaved band at $80 \mathrm{Kda}$ compared with the controls (Figure 2A). Notch 1 is a transmembrane domain protein that is involved in the development and determination of cell fate [17]. During maturation, the Notch 







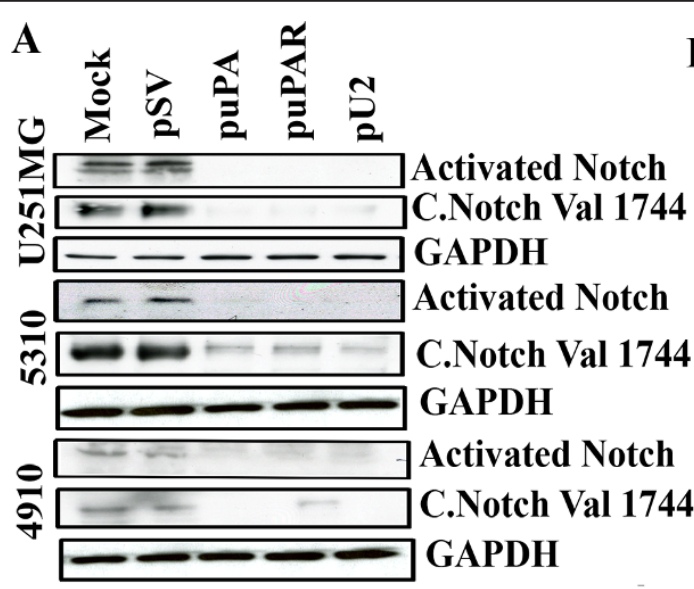

B

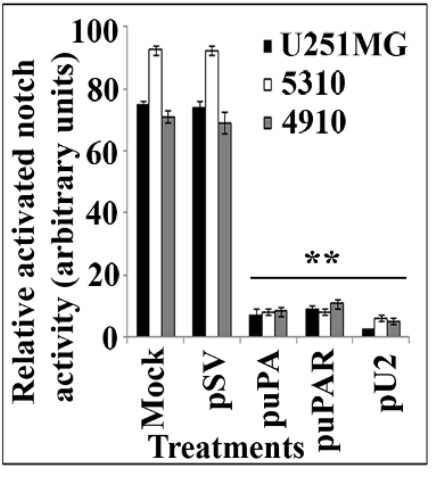

C

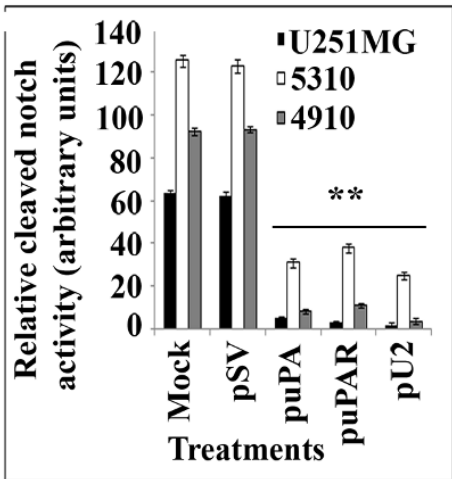

D



$\mathbf{E}$
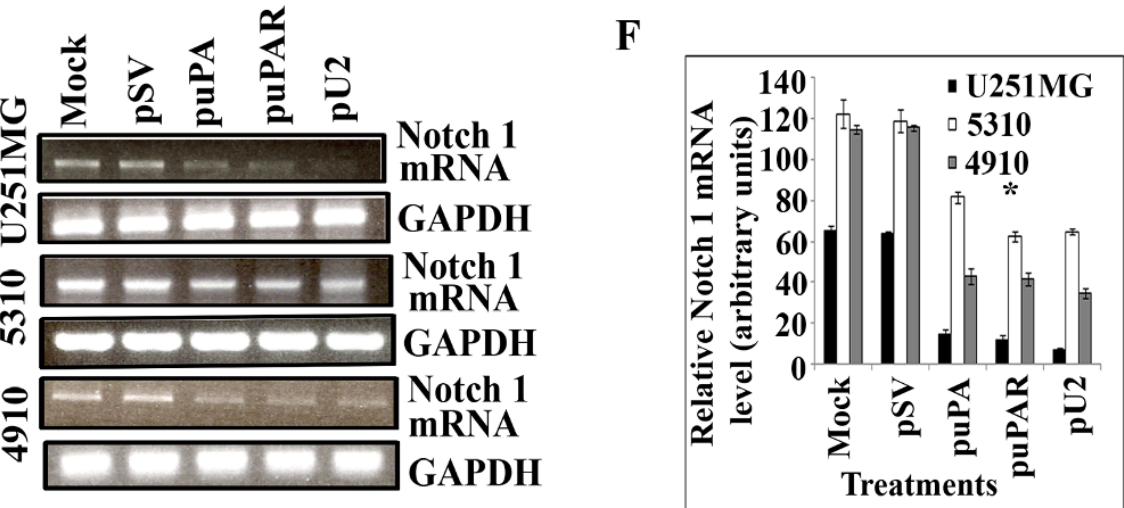

Figure 2 Transcriptional inactivation of uPA and/or uPAR by shRNA inhibits cleavage of Notch 1 receptor, Notch 1 gene expression in U251 MG, 4910 and 5310 xenograft cells. (A) Whole cell lysates were prepared from U251 MG, 4910 and 5310 cells which were left untransfected, or transfected with pSV, puPA, puPAR and pU2 to evaluate the protein levels of activated Notch 1 and for Notch 1 cleavage at the Val 1744 position. Blots were probed with GAPDH antibody to analyze for equal loading of proteins. (B) \& (C) Quantitative analysis of relative Notch 1 expression and cleaved Notch expression in U251 MG, 5310 and 4910 cells. (D) Immunofluorescence analysis of U251MG cells left untransfected or transfected with pSV, puPA, puPAR and pU2 and stained for NICD (Notch intracellular domain) and images taken with Olympus Fluoview microscope. (E) RT-PCR analysis of Notch 1 mRNA expression in (a) U251 MG, 5310 and 4910 cells which were left untransfected or transfected with pSV, puPA, puPAR and pU2. GAPDH was used as an internal control. (F) Quantitative representation of Notch 1 mRNA levels in U251MG, 5310 and 4910 cells. Values are mean \pm S.D. of three independent experiments $\left({ }^{*} P<0.01,{ }^{* *} P<0.001\right)$. 
molecule is cleaved by a furin-like convertase at its extracellular domain [18]. Upon ligand binding, the carboxy terminal Notch 1 fragment is released and is further cleaved at Gly 1743 and Val 1744 [19,20]. Endogenous levels of cytosolic domain are observed only upon cleavage between Gly 1743 and Val 1744. Results show cleavage of Notch at the Val 1744 position is inhibited in puPA- and pU2-transfected U251, 4910 and 5310 cells (Figure 2A). Quantitative representation of activated Notch 1 and cleaved Notch 1 levels is shown in Figure 2B and Figure 2C, respectively. To further confirm our western blotting results, we checked for the NICD domain in U251 MG cells by staining the cells with an antibody to NICD in untreated U251 MG cells and in U251 MG cells transfected with pSV, puPA, puPAR and pU2. As demonstrated by our western blot results, we observed increased expression of NICD in mock and pSV-treated cells (Figure 2D, panel a \& b) while the cells treated with the three shRNA constructs showed very little staining (Figure 2D, panel c to e). NICD staining was seen as small green spots at the periphery and at the center of the cells.

pUPA, pUPAR and pU2 inhibits the gene expression of Notch 1 mRNA in all the three cell lines examined

To determine whether puPA, puPAR and pU2 inhibit Notch 1 mRNA, we subjected controls and shRNAtransfected U251 MG, 5310 and 4910 cells to RT PCR for Notch 1 gene expression. Results showed that simultaneous down regulation of UPA and UPAR by pU2 efficiently down regulated Notch $1 \mathrm{mRNA}$ in all three cell lines compared with puPA and puPAR (Figure 2E). Quantitative analysis of the RT-PCR results is shown in Figure 2F. These results suggest that simultaneous down regulation of both uPA and uPAR with pU2 is a better approach to knockdown Notch 1 mRNA than using single shRNA constructs.

\section{Notch inhibits uPA expression and activity in glioblastoma cells and xenografts}

To ascertain whether Notch plays a role in regulating uPA activity and expression, we transfected U251 MG, 5310 and 4910 cells with scrambled vector siRNA and siRNA to Notch 1 and checked for uPA activity by fibrinogen zymography and uPA expression using western blotting. In all three cell lines tested, results showed that Notch siRNA downregulated uPA expression (Figure 3A) and uPA activity (Figure 3B). This probably suggests that Notch and uPA regulate each other through a positive feedback mechanism. Quantitative expression of relative uPA expression and relative $\mathrm{UPA}$ activity is shown in Figures $3 \mathrm{C}$ and Figure $3 \mathrm{D}$, respectively.
Exogenous addition of UPA and UPAR activate Notch 1 in UPAR antisense and in U251 MG cells

Our previous results showed that shRNA against uPA and UPAR inhibits Notch expression and cleavage (Figure 2A). We wanted to check if addition of exogenous UPA and UPAR activates the Notch 1 receptor in UPAR antisense cells and in U251 MG cells. Our results indicate that upon exogenous addition of uPA and ruPAR, individually and in combination, Notch 1 expression was activated in UPAR antisense cells and in U251 MG cells (Figure 3E). This probably suggests that uPA independent of its role as a UPAR ligand can activate the Notch 1 receptor in glioblastoma cells.

\section{uPA activates Notch 1 receptor independent of Notch ligands, Delta and Jagged}

To further clarify the role of uPA as a ligand for Notch 1 in glioblastoma cells, we wanted to ascertain if uPA activates Notch 1 in uPAR antisense cells in both the presence and absence of Notch ligands, namely Delta and Jagged. For this purpose, we used siDelta and siJagged transfected UPAR antisense cells and stimulated these cells with uPA. Surprisingly, we found that in uPAR antisense cells, exogenous uPA activated Notch 1 and showed a further increase in fold activation in the presence of siDelta and Jagged siRNA (Figure 3F). These results suggest that Notch 1 can be activated by uPA by independent of uPAR and Notch ligands, Delta and Jagged. Quantitative expression of Notch 1 expression from Figure $3 \mathrm{E} \& 3 \mathrm{~F}$ is depicted in Figure $3 \mathrm{G}$ and Figure $3 \mathrm{H}$, respectively. Overall, these results indicate that Notch 1 and uPA regulate each other in glioblastoma and GBM uPAR antisense cells.

\section{puPA, puPAR and pU2 inhibits NICD and HES-induced} AKT, NF- $\kappa$ B and ERK pathways in U251 MG and 4910 cells Previously, we have shown that down regulation of uPA, UPAR and pU2 in SNB19 cells leads to decreased phosphorylation of AKT and mTOR at the Ser 2448 position, and ERK downstream of AKT. To further confirm these results in the cells lines used in this study, we checked for phosphorylation of AKT, ERK and mTOR after transfection with shRNA against uPA/uPAR, singly and in combination. As anticipated, we observed that transfection with puPA-, puPAR- and pU2-transfected cells significantly down regulated phosphorylation of AKT, mTOR and ERK pathways in the three cell lines used in this study. (Figure 4A-C). Previous studies have shown that NF- $\kappa \mathrm{B}$ is a downstream target for ERK [21]. To confirm whether uPA/uPAR downregulation inhibits $\mathrm{NF}-\kappa \mathrm{B}$, which is downstream to ERK, we checked the nuclear activation of p50 (NF- $\kappa \mathrm{B} 1), \mathrm{p} 52(\mathrm{NF}-\kappa \mathrm{B} 2)$ and p65 (Rel A). As expected, we observed significant reduction in the nuclear activation of p50, p52 and p65 in all 



D
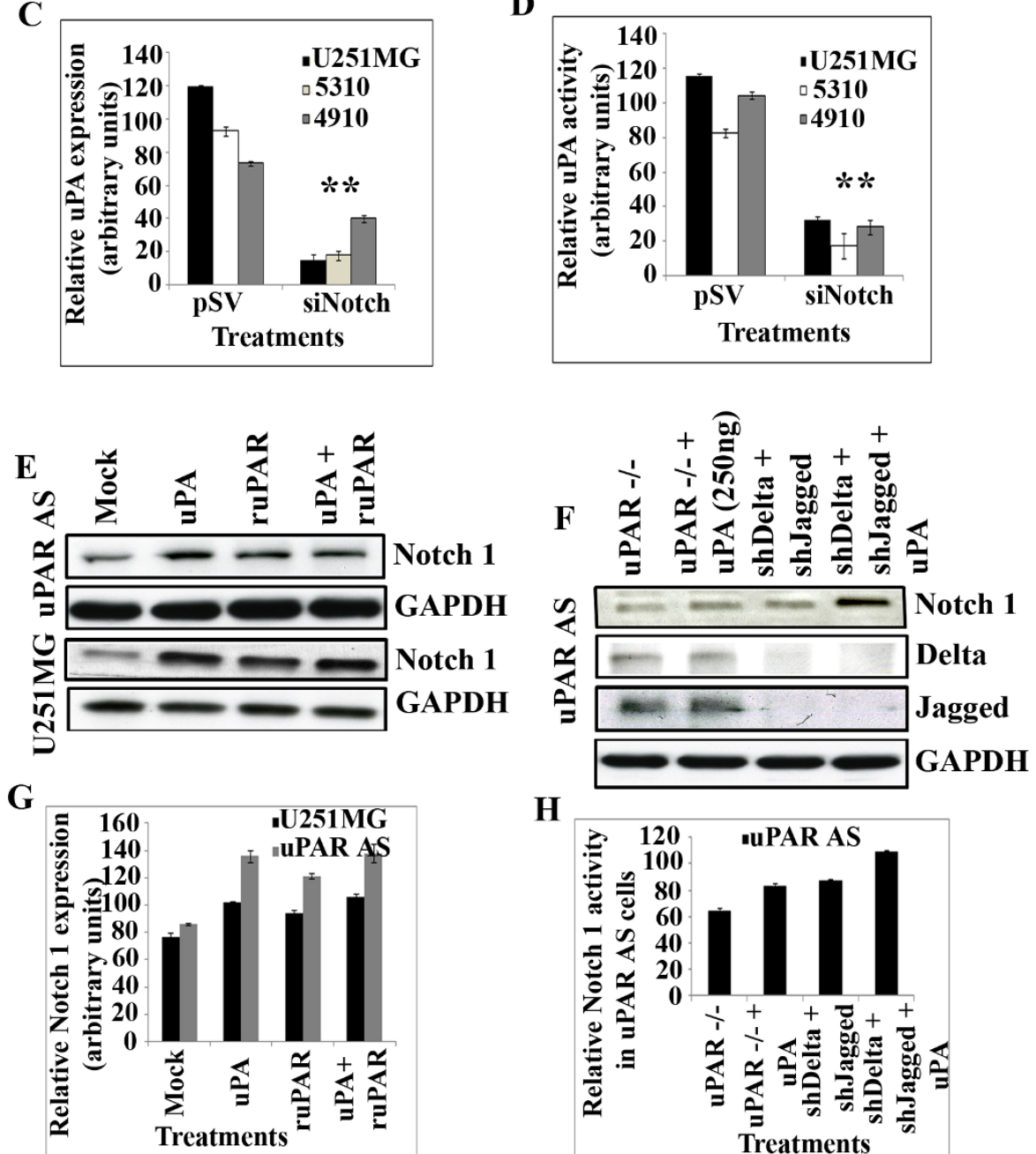

$\mathbf{H}$

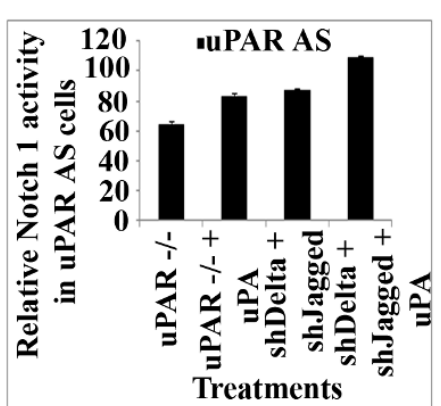

Figure 3 uPA positively regulates Notch in glioblastoma cells. (A) U251 MG, 5310 and 4910 cells were transfected with pSV (scrambled Vector) or with siRNA against Notch 1 and analyzed for uPA and Notch 1 expression by western blotting. Blots were probed with GAPDH antibody to analyze for equal loading of proteins. (B) Fibrin zymography of U251 MG, 5310 and 4910 cells transfected with pSV (scrambled Vector) and siNotch 1 was performed to analyze for UPA activity. (C) \& (D) Quantitative expression of relative uPA expression and uPA activity in pSV and siNotch-transfected cells. (E) Exogenous addition of purified uPA activates Notch 1 receptor in uPAR antisense cells and in U251 MG cells. Whole cell lysates prepared from UPAR antisense and U251 MG cells, which were treated with purified uPA (250 ng), ruPAR (50 ng) and in combination, were checked for Notch 1 expression by Western blotting analysis. Blots were probed with GAPDH antibody to analyze for equal loading of lysates. (F) Whole cell lysates prepared from UPAR antisense cells which were left untreated, treated with purified uPA, transfected with shRNA against Delta and Jagged (Notch 1 ligands) with and without stimulation with purified uPA and probed for Notch 1, Delta and Jagged by Western blotting. Blots were probed with GAPDH antibody to analyze for equal loading of lysates. (G) Quantitative analysis of relative Notch 1 expression represented in (E) and (H) Quantitative analysis of relative Notch 1 expression represented in (F). Values are mean \pm S.D. of three independent experiments in comparison with the controls $\left({ }^{*} \mathrm{P}<0 / 01,{ }^{* *} \mathrm{P}<0.001\right)$. 


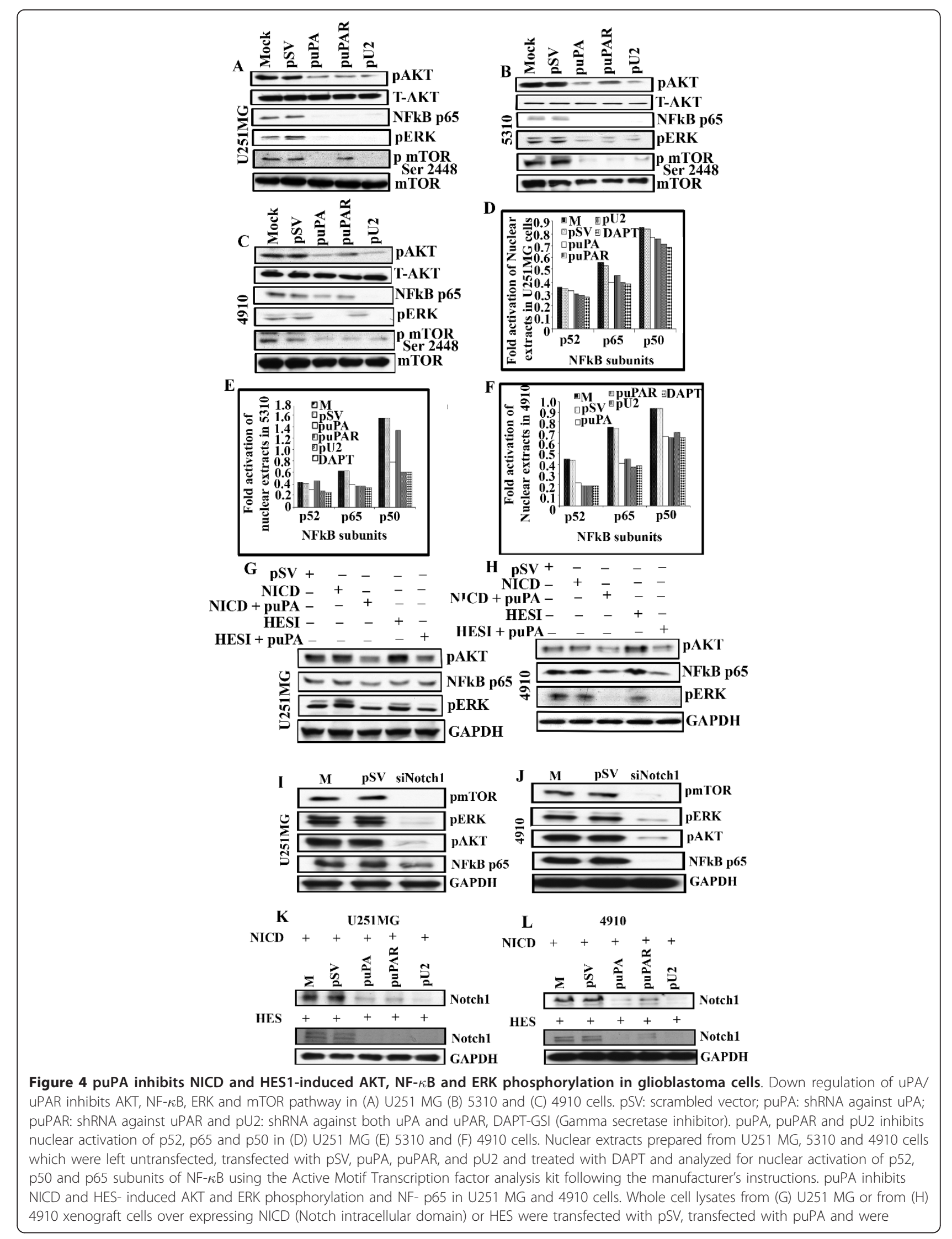


immunoblotted for pAKT, NF- $\kappa$ B p65 and pERK. Blots were reprobed with GAPDH to analyze for equal loading of proteins. Data represents average of triplicates normalized to GAPDH (** $<0.01$ ). (I \& J) siRNA against Notch 1 inhibits phosphorylated forms of mTOR/ERK/AKT and NF$\kappa B$ p65 in (I) U251MG and (J) 4910 cells. Whole cell lysates from U251MG and 4910 cells were left untreated, transfected with pSV and siNotch for 48 hrs and were immunoblotted for pmTOR, pERK, pAKT and NF- $\kappa$ B p65. A representative blot of three independent experiments is shown. Blots were reprobed with e GAPDH to analyze for equal loading of proteins. (K\&L) shRNA against UPA, UPAR and U2 inhibited NICD/HES1 induced Notch 1 expression in U251MG and 4910 cells. (K) Whole cell lysates from (G) U251 MG or from (H) 4910 xenograft cells over expressing NICD (Notch intracellular domain) or HES were transfected with pSV, puPA, puPAR and pU2 and were immunoblotted for Notch 1 expression. A representative blot of three independent experiments is shown. Blots were reprobed with GAPDH to analyze for equal loading of proteins.

three cell lines tested (Figure 4D-F). Overall, these results suggest that shRNA against $\mathrm{UPA}$, UPAR and U2 inhibits AKT/mTOR, ERK and NF- $\kappa$ B pathways in glioblastoma cells and xenografts.

The cross talk between Notch signaling and AKT/ mTOR pathways has been reported in the literature in many studies [22-24]. Previous reports in the literature $[25,26]$ have shown that there is a cross talk between Notch and NF- $\kappa$ B. To determine whether puPA down regulates Notch-induced AKT, NF- $\kappa \mathrm{B}$ and ERK pathways, we overexpressed NICD and HES1 in U251 MG and 4910 cells. We also transfected these cells with puPA to check if puPA inhibits NICD and HES-induced phosphorylation of $\mathrm{AKT}, \mathrm{NF}-\kappa \mathrm{B}$ and phosphorylation of ERK. Interestingly, we observed activation of AKT, ERK and NF- $\kappa$ B in pSVtransfected cells and in NICD and HES1-overexpressed cells (Figure 4G and 4H). AKT, ERK phosphorylation and NF-kB p65 levels were significantly down regulated upon transfection with puPA (Figure 4G and 4H). To confirm the cross talk of AKT/mTOR/NF- $\kappa \mathrm{B} / \mathrm{ERK}$ pathways with Notch-1, U251MG and 4910 cells were left untreated or treated with pSV and siNotch 1 and checked for the expression of pmTOR, pERK, pAKT and NF-kB p65. Results showed that siNotch 1 inhibited phosphorylation of AKT, ERK, mTOR and NF-kB levels compared with controls in both the cell lines tested (Figure 4I and Figure $4 \mathrm{~J})$. To further confirm if NICD/HES1-overexpressed Notch 1 is inhibited by puPA, puPAR and pU2 shRNA constructs, we checked for Notch 1 expression in U251MG and 4910 cells after transfection with shRNA constructs against uPA, uPAR and U2. Results showed that NICD/HES1 induced Notch1 expression levels were significantly reduced in UPA andU2 transfected cells and moderately in puPAR transfected cells (Figure 4K and Figure $4 \mathrm{~L}$ ). These results suggest that shRNA against uPA, uPAR and U2 inhibit AKT, ERK and NF- $\kappa$ B pathways. These results also show that shRNA against uPA down regulates NICD and HES-induced Notch1, AKT, NF- $\kappa$ B and ERK activation in glioblastoma cell lines.

\section{Transcriptional inactivation of UPA/UPAR inhibits trafficking of Notch 1 receptor in the U251 MG cell line} The trafficking of Notch receptor is known to be mediated by endosomes. To determine if Notch 1 is targeted to lysosomes after transfection with shRNA against uPA, uPAR and U2 (bicistronic construct), we conducted immunofluorescent staining in U251 cells after transfection and checked for co-localization of NICD with LAMP-1 (a lysosomal marker). We did not observe co-localization of Notch 1 with LAMP-1 in mock and pSV-treated cells (Figure 5A, panel a to f) but there were few spots of co-localization in puPA- (Figure $5 \mathrm{~A}$, panel $\mathrm{g}$ to $\mathrm{i}$ ) and puPAR- (Figure $5 \mathrm{~A}$, panel $\mathrm{j}$ to $\mathrm{l}$ ) treated cells. In pU2-treated cells, we were able to observe a significant association of Notch 1 receptor with LAMP-1, as indicated by a significant number of yellow spots which is suggestive of co-localization (Figure 5A, panel $\mathrm{m}$ to o). The percent of cell count showing co-localization is depicted quantitatively in Figure $5 \mathrm{~B}$. This result suggests that Notch 1 receptor trafficking is significantly affected in puPA- puPAR- and pU2transfected U251 MG cells.

\section{puPA and pU2 affect growth and pre-established intracranial tumors in nude mice}

To further confirm our in vitro results, we checked if shRNA against puPA and pU2 affected pre-established intracranial tumors in nude mice and also assessed Notch 1 expression. In U251 MG pre-established intracranial tumors, we observed expression of Notch 1 (Figure $6 \mathrm{~A}$, panel a to $\mathrm{c}$ ), but in puPA-treated tumors, we observed a significant decrease in the tumor margin and infiltration, as well as in inhibition of Notch 1 expression (Figure 6A, panel $\mathrm{d}$ to $\mathrm{f}$ ). In pU2-treated tumors, the tumor margin showed more inhibition than in puPA-treated tumors, and there was also significant inhibition of Notch 1 expression and more inhibition at the tumor margin than in puPA- treated tumors. (Figure $6 \mathrm{~A}$, panel g to i). Overall, these results show that uPA and UPAR down regulation in combination inhibits invasion in glioblastoma cells by down regulation of Notch 1 receptor activation and expression, as well as by blocking the trafficking of the Notch 1 receptor.

Expression of Notch 1 has been reported in various types of human cancers $[27,28]$. Notch 1 has shown to be involved in several functions in cancer cells including proliferation, invasion and angiogenesis $[21,29,30]$. In this study, we report that Notch 1 is over expressed in 


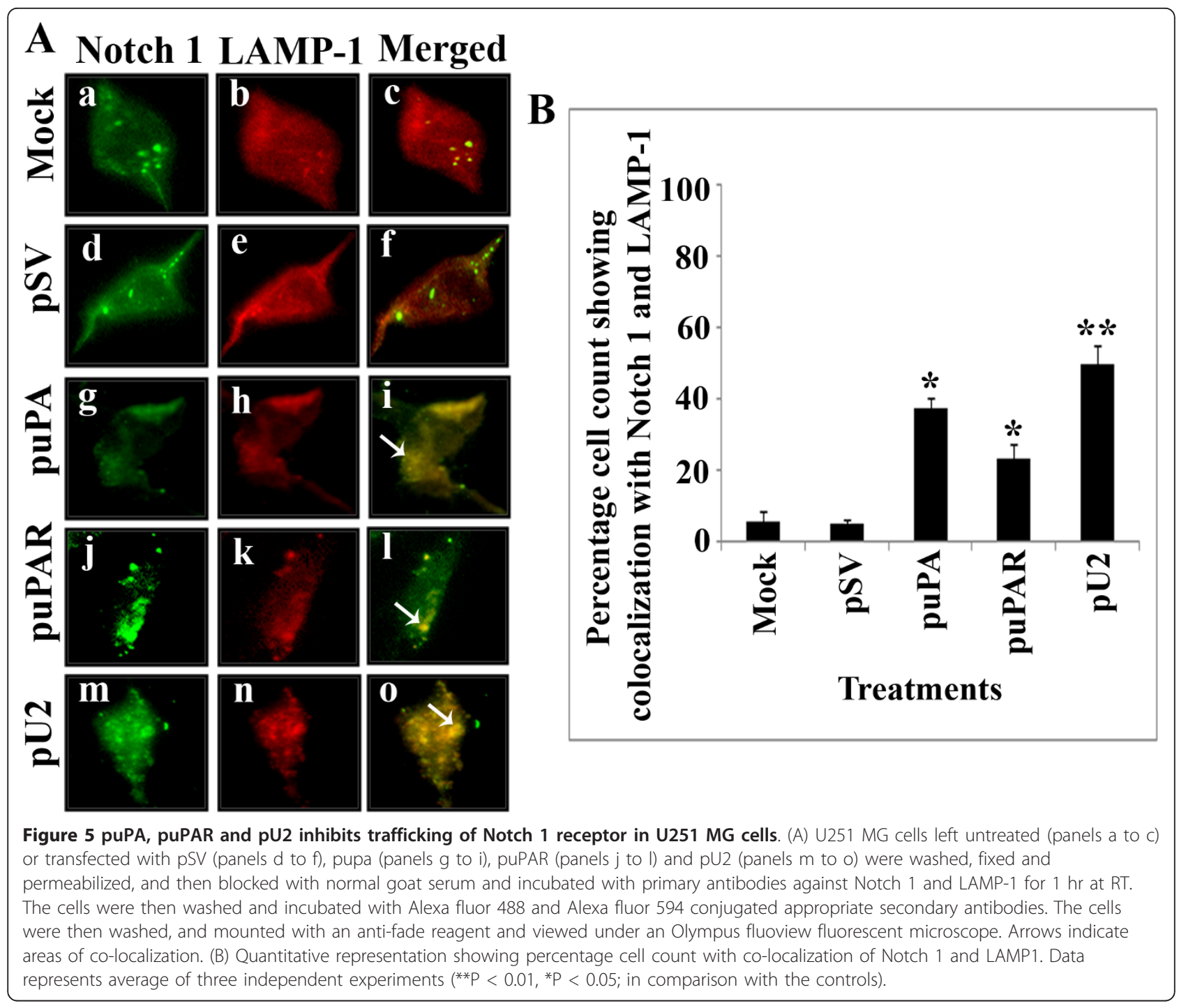

glioblastoma cell line U251 MG and xenografts consistent with previous studies $[11,12,31]$. Studies by us and others have previously shown that simultaneous down regulation of UPA and UPAR in glioblastoma cells significantly down regulated invasion and angiogenesis by in vitro and in vivo [32,33]. In this study, we found that Notch 1 is a direct target for puPA and by pU2. Studies by many groups $[11,34,35]$ have shown that Notch 1 is target for many genes in glioblastoma, and siRNA against Notch 1 down regulates uPA and inhibits prostate cancer and pancreatic cancer cell invasion $[27,36]$. Inspite of this current knowledge, the role of UPA and UPAR down regulation in glioma cell invasion remains poorly understood. In this study, we show that knockdown of uPA and $\mathrm{UPAR}$ in tandem inhibits cleavage of the Notch receptor, down regulates Notch 1 gene expression and Notch 1 signaling cross talk, and significantly blocks the trafficking of the Notch receptor in glioblastoma cells.
As previously shown in glioblastoma cell line SNB19, we observed that targeted down regulation of UPA and UPAR by single and bicistronic constructs inhibited invasion in U251 MG and in glioma xenografts 4910 and 5310 by Matrigel invasion assay (Figure 1F) and also anchorage independent growth by soft agar colony formation assay (Figure 1D). Increased expression of Notch 1 has been detected in brain tumors [37] and other cancers [38,39]. The role of Notch 1 in different cancers is considerably varied [40]. As previously reported [11], we observed increased expression of Notch 1 in glioma human tissue array, western blot and RT-PCR.

The down regulation of UPA and UPAR inhibited activated Notch 1 activity and cleavage of Notch 1 at the Val 1744 position which resulted in the inhibition of Notch gene transcription. Moreover, glioblastoma cells treated with siRNA against Notch showed decreased 


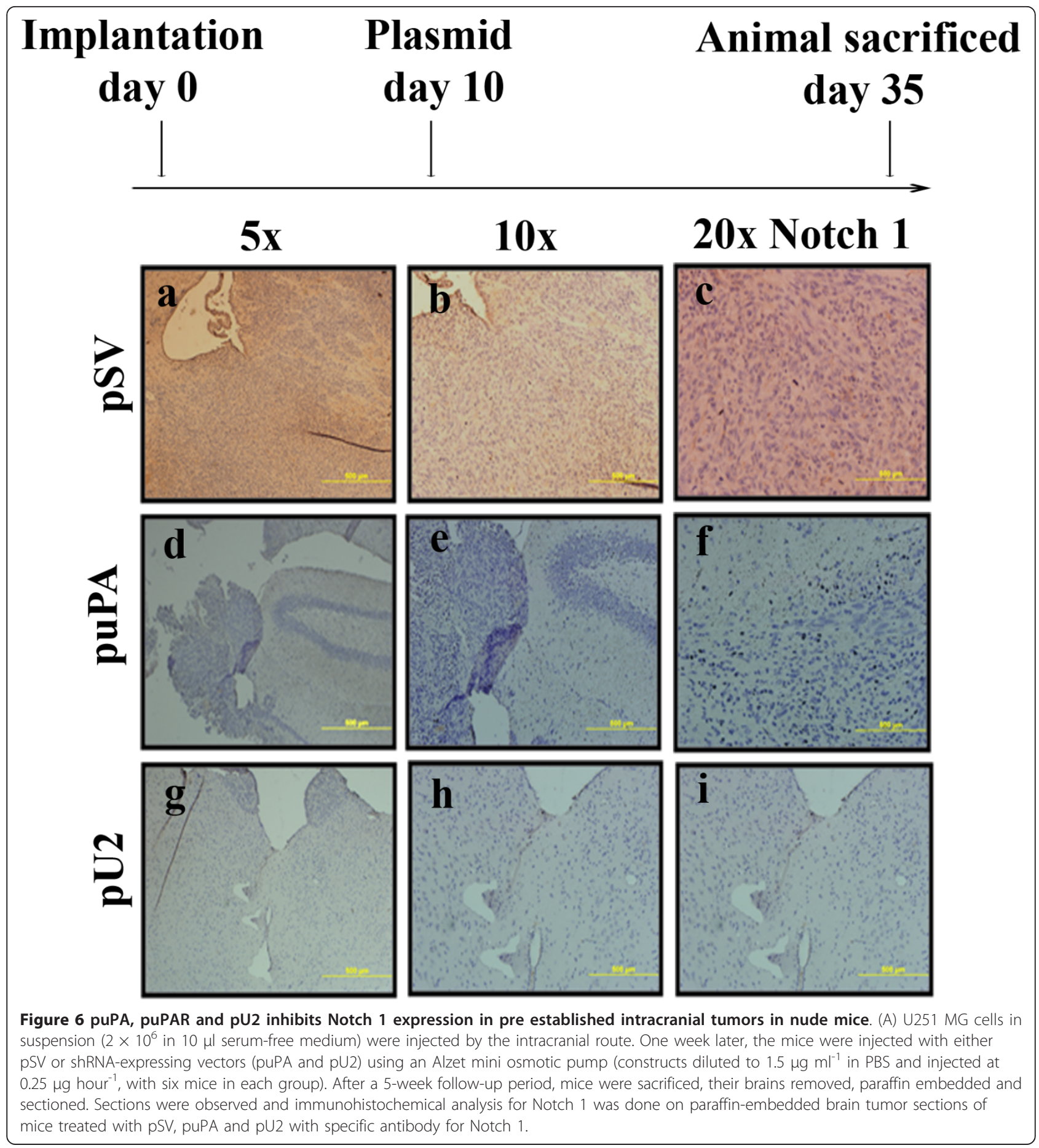

uPA expression as shown by western blotting and decreased uPA activity by fibrin zymography. Based on these observations, it is tempting to speculate that the uPA/uPAR system might play a direct role in Notch 1 signaling and that $\mathrm{UPA}$ alone could directly regulate Notch 1 in glioblastoma. Whether uPA/uPAR regulates Notch 1 promoter activity is to be studied in future.
Our data corresponds to a previous study [41] which showed that Notch 1 knockdown regulates the expression of uPA and UPAR by a distinct gene expression mechanism in prostrate cancer cells. Bin Hafeez et al. [41] conclude their study by stating that in addition to MMP-9, uPA and UPAR might work synergistically to enhance tumor invasion and metastasis in prostrate 
cancer. As our results show that Notch 1 expression is severely altered with puPA, puPAR and pU2 treatments, we hypothesize that uPA/uPAR could be a target for intervention in glioma.

Previous studies have shown that Notch 1 regulates the PI3K/AKT/mTOR pathway in other cancers. Notch 1 has been reported to cross talk with the AKT pathway in human cancer cells [22-24,42]. Studies by us have also shown that UPA and UPAR down regulation inhibits phosphorylation of Akt [32] in glioblastoma cells. Our results suggest that inhibition of AKT/mTOR signaling pathway via uPA/uPAR down regulation might be mediated by Notch 1 signaling in glioblastoma cells. Phosphorylation of AKT induced by NICD and HES 1 over expression was down regulated by shRNA against uPA in GBM cells. These results suggest the existence of a reciprocal regulation of Notch-1 and AKT pathway in uPA/uPAR down regulation in glioblastoma cells.

Notch is known to cross talk with NF- $\kappa$ B and ERK pathways which are downstream of AKT $[27,36]$. Our studies have shown that UPA/uPAR down regulation inhibits nuclear activation of NF- $\kappa$ B [43] and phosphorylation of ERK [41]. The present study has also shown that NF- $\kappa B$ and AKT induced by NICD and HES1 over expression in glioblastoma cell lines is inhibited by shRNA against uPA. Further in-depth mechanistic studies are required to understand the role of $\mathrm{uPA}$ in regulation of Notch 1-induced AKT, NF- $\kappa \mathrm{B}$ and ERK pathways. Our results have comprehensively suggested that $\mathrm{UPA} / \mathrm{UPAR}$ down regulation results in the inhibition of invasion of GBM cells by targeting the Notch 1 receptor signaling, cleavage, decreased Notch1 mRNA transcription and trafficking. Purow et al [11] have shown that Notch siRNA leads to increased cell death, decreased proliferation and inhibition of cell cycle in glioblastoma cells. Our results suggest that uPA/uPAR down regulation might regulate Notch 1-mediated events in glioblastoma. Interestingly, our results show that $\mathrm{UPA} / \mathrm{uPAR}$ down regulation resulted in Delta and Jagged downregulation (data not shown). Studies by Purow et al [11] have also shown that Notch ligands could themselves have a potential for transformation and play important roles in gliomagenesis. How shRNA against uPA and uPAR regulates Delta and Jagged is under further investigation. Our results also show that in uPAR antisense cells and in U251 MG cells, UPA was able to activate Notch 1 in the absence of Delta and Jagged ligands. These results suggest that uPA by itself could mediate UPAR independent functions such as Notch 1 regulation in glioblastoma cell lines.

Our studies also indicate that UPA/UPAR down regulation also results in the lysosomal targeting of the Notch 1 receptor in U251 MG cells. The mechanistic details of lysosomal targeting are yet to be explored.
Notch 1 expression was also inhibited in pupa- and pU2-treated pre-established intracranial tumors in nude mice. Involvement of uPA/uPAR in a plethora of cellular processes in glioma gives us the advantage of targeting this multifunctional system for therapeutic intervention in patients with high grade glioma.

\section{Conclusions}

In summary, we have presented experimental evidence that strongly suggests the role of Notch 1 in uPA/uPAR down regulation in glioblastoma. shRNA against uPA and UPAR inhibited Notch 1 cleavage, Notch gene transcription and Notch 1-mediated AKT/ERK phosphorylation and NF- $\kappa$ B subunits. From our results, we can conclude that down regulation of uPA/uPAR, singly and in tandem, could be an effective therapeutic approach for inactivation of Notch-1 cleavage, signaling and trafficking and to down regulate Notch-signaling-induced NF- $\kappa \mathrm{B}, \mathrm{ERK}$ and AKT pathways which are known to play roles in growth, migration, invasion and angiogenesis of glioblastoma. Based on our results, we provide a hypothetical pathway by which uPA/uPAR inhibits the Notch 1 pathway which may be partly mediated by AKT/NF- $\kappa \mathrm{B}$ and ERK pathways. Overall, our results suggest that targeting uPA/uPAR may be advantageous for future treatment of gliomas.

\section{Funding}

This research was supported by a grant from National Institutes of Health, CA75557 (to J.S.R.). The contents are solely the responsibility of the authors and do not necessarily represent the official views of NIH. The funders had no role in study design, data collection and analysis, decision to publish, or preparation of the manuscript.

\section{List of Abbreviations}

UPA: urokinase plasminogen activator; UPAR: urokinase plasminogen activator receptor; AKT: RAC-alpha serine/threonine-protein kinase; PI3-K: phosphotidyl inositide 3 kinase; ERK:Extracellular regulated kinase; NICD: Notch intracellular domain: HES: Hairy enhancer of split; GAPDH: glyceraldehydephosphate 3 dehydrogenase; LAMP-1: Iysosome associated membrane protein 1.

\section{Acknowledgements}

The authors thank Shellee Abraham for manuscript preparation and Diana Meister and Sushma Jasti for manuscript review.

\section{Author details}

'Department of Cancer Biology and Pharmacology, University of Illinois College of Medicine, One Illini Drive, Peoria, IL 61605, USA. ${ }^{2}$ Department of Neurosurgery, University of Illinois College of Medicine, One Illini Drive, Peoria, IL 61605, USA. ${ }^{3}$ Department of Pathology, University of Illinois College of Medicine, One Illini Drive, Peoria, IL 61605, USA.

\section{Authors' contributions}

$H R$ conceived and carried out the experiments. HR wrote the manuscript. $H R, C G$ and JSR reviewed and analyzed the data. JSR, MG and DD 
contributed reagents/materials/analysis tools. All members read and approved the final manuscript.

\section{Competing interests}

The authors declare there are no conflicts of interest regarding this manuscript.

Received: 13 June 2011 Accepted: 17 October 2011

Published: 17 October 2011

\section{References}

1. Wang Z, Li Y, Banerjee S, Sarkar FH: Exploitation of the Notch signaling pathway as a novel target for cancer therapy. Anticancer Res 2008, 28:3621-3630.

2. Leong $K G$, Karsan $A$ : Recent insights into the role of Notch signaling in tumorigenesis. Blood 2006, 107:2223-2233.

3. Rehman $\mathrm{AO}$, Wang $\mathrm{CY}$ : Notch signaling in the regulation of tumor angiogenesis. Trends Cell Biol 2006, 16:293-300.

4. Poellinger $L$, Lendahl U: Modulating Notch signaling by pathway-intrinsic and pathway-extrinsic mechanisms. Curr Opin Genet Dev 2008, 18:449-454.

5. Fischer I, Gagner JP, Law M, Newcomb EW, Zagzag D: Angiogenesis in gliomas: biology and molecular pathophysiology. Brain Pathol 2005, 15:297-310.

6. Miele L: Notch signaling. Clin Cancer Res 2006, 12:1074-1079.

7. Xu P, Qiu M, Zhang Z, Kang C, Jiang R, Jia Z, Wang G, Jiang H, Pu P: The oncogenic roles of Notch1 in astrocytic gliomas in vitro and in vivo. $J$ Neurooncol 2010, 97:41-51.

8. Guo D, Ye J, Dai J, Li L, Chen F, Ma D, Ji C: Notch-1 regulates Akt signaling pathway and the expression of cell cycle regulatory proteins cyclin D1, CDK2 and p21 in T-ALL cell lines. Leuk Res 2009, 33:678-685.

9. Rao SS, O'Neil J, Liberator CD, Hardwick JS, Dai X, Zhang T, Tyminski E, Yuan J, Kohl NE, Richon VM, Van der Ploeg LH, Carroll PM, Draetta GF, Look AT, Strack PR, Winter CG: Inhibition of NOTCH signaling by gamma secretase inhibitor engages the RB pathway and elicits cell cycle exit in T-cell acute lymphoblastic leukemia cells. Cancer Res 2009, 69:3060-3068.

10. Gondi CS, Kandhukuri N, Dinh DH, Gujrati M, Rao JS: Down-regulation of UPAR and UPA activates caspase-mediated apoptosis and inhibits the PI3K/AKT pathway. Int J Oncol 2007, 31:19-27.

11. Purow BW, Haque RM, Noel MW, Su Q, Burdick MJ, Lee J, Sundaresan T, Pastorino S, Park JK, Mikolaenko I, Maric D, Eberhart CG, Fine HA: Expression of Notch-1 and its ligands, Delta-like- 1 and Jagged-1, is critical for glioma cell survival and proliferation. Cancer Res 2005, 65:2353-2363.

12. Kanamori M, Kawaguchi $T$, Nigro JM, Feuerstein BG, Berger MS, Miele L Pieper RO: Contribution of Notch signaling activation to human glioblastoma multiforme. J Neurosurg 2007, 106:417-427.

13. Shaik S, Hirao H, Kumar D: Reactivity patterns of cytochrome P450 enzymes: multifunctionality of the active species, and the two statestwo oxidants conundrum. Nat Prod Rep 2007, 24:533-552.

14. Mohanam S, Jasti SL, Kondraganti SR, Chandrasekar N, Kin Y, Fuller GN, Lakka SS, Kyritsis AP, Dinh DH, Olivero WC, Gujrati M, Yung WK, Rao JS: Stable transfection of urokinase-type plasminogen activator antisense construct modulates invasion of human glioblastoma cells. Clin Cancer Res 2001, 7:2519-2526.

15. Koleske AJ, Baltimore D, Lisanti MP: Reduction of caveolin and caveolae in oncogenically transformed cells. Proc Natl Acad Sci USA 1995, 92:1381-1385

16. Nalla AK, Gorantla B, Gondi CS, Lakka SS, Rao JS: Targeting MMP-9, uPAR, and cathepsin B inhibits invasion, migration and activates apoptosis in prostate cancer cells. Cancer Gene Ther 2010, 17:599-613.

17. Artavanis-Tsakonas S, Rand MD, Lake RJ: Notch signaling: cell fate control and signal integration in development. Science 1999, 284:770-776.

18. Chan YM, Jan YN: Roles for proteolysis and trafficking in notch maturation and signal transduction. Cell 1998, 94:423-426.

19. Schroeter EH, Kisslinger JA, Kopan R: Notch-1 signalling requires ligandinduced proteolytic release of intracellular domain. Nature 1998, 393:382-386.

20. Rand MD, Grimm LM, rtavanis-Tsakonas S, Patriub V, Blacklow SC, Sklar J, Aster JC: Calcium depletion dissociates and activates heterodimeric notch receptors. Mol Cell Biol 2000, 20:1825-1835.
21. Zeng Q, Li S, Chepeha DB, Giordano TJ, Li J, Zhang H, Polverini PJ, Nor J, Kitajewski J, Wang CY: Crosstalk between tumor and endothelial cells promotes tumor angiogenesis by MAPK activation of Notch signaling. Cancer Cell 2005, 8:13-23.

22. Gutierrez A, Look AT: NOTCH and PI3K-AKT pathways intertwined. Cancer Cell 2007, 12:411-413.

23. Palomero T, Sulis ML, Cortina M, Real PJ, Barnes K, Ciofani M, Caparros E, Buteau J, Brown K, Perkins SL, Bhagat G, Agarwal AM, Basso G, Castillo M, Nagase S, Cordon-Cardo C, Parsons R, Zuniga-Pflucker JC, Dominguez M, Ferrando AA: Mutational loss of PTEN induces resistance to NOTCH1 inhibition in T-cell leukemia. Nat Med 2007, 13:1203-1210.

24. Bedogni B, Warneke JA, Nickoloff BJ, Giaccia AJ, Powell MB: Notch1 is an effector of Akt and hypoxia in melanoma development. $J$ Clin Invest 2008, 118:3660-3670

25. Osipo C, Golde TE, Osborne BA, Miele LA: Off the beaten pathway: the complex cross talk between Notch and NF-kappaB. Lab Invest 2008, 88:11-17.

26. Song R, Kim YW, Koo BK, Jeong HW, Yoon MJ, Yoon KJ, Jun DJ, Im SK, Shin J, Kong MP, Kim KT, Yoon K, Kong YY: Mind bomb 1 in the lymphopoietic niches is essential for $T$ and marginal zone $B$ cell development. J Exp Med 2008, 205:2525-2536.

27. Wang Z, Li Y, Banerjee S, Kong D, Ahmad A, Nogueira V, Hay N, Sarkar FH Down-regulation of Notch-1 and Jagged-1 inhibits prostate cancer cell growth, migration and invasion, and induces apoptosis via inactivation of Akt, mTOR, and NF-kappaB signaling pathways. J Cell Biochem 2010, 109:726-736

28. Koch U, Radtke F: Notch and cancer: a double-edged sword. Cell Mol Life Sci 2007, 64:2746-2762.

29. Hellstrom M, Phng LK, Hofmann JJ, Wallgard E, Coultas L, Lindblom P, Alva J, Nilsson AK, Karlsson L, Gaiano N, Yoon K, Rossant J, Iruela-Arispe ML, Kalen M, Gerhardt H, Betsholtz C: DIl4 signalling through Notch1 regulates formation of tip cells during angiogenesis. Nature 2007, 445:776-780.

30. Noguera-Troise I, Daly C, Papadopoulos NJ, Coetzee S, Boland P, Gale NW, Lin HC, Yancopoulos GD, Thurston G: Blockade of Dll4 inhibits tumour growth by promoting non-productive angiogenesis. Nature 2006, 444:1032-1037.

31. Stockhausen MT, Kristoffersen K, Poulsen HS: The functional role of Notch signaling in human gliomas. Neuro Oncol 2010, 12:199-211.

32. Gondi CS, Lakka SS, Dinh DH, Olivero WC, Gujrati M, Rao JS: Intraperitoneal injection of an hpRNA-expressing plasmid targeting UPAR and UPA retards angiogenesis and inhibits intracranial tumor growth in nude mice. Clin Cancer Res 2007, 13:4051-4060.

33. Raghu H, Lakka SS, Gondi CS, Mohanam S, Dinh DH, Gujrati M, Rao JS Suppression of UPA and UPAR attenuates angiogenin mediated angiogenesis in endothelial and glioblastoma cell lines. PLoS One 2010, 5 e12458.

34. Guessous F, Li Y, Abounader R: Signaling pathways in medulloblastoma. J Cell Physiol 2008, 217:577-583

35. Li Y, Guessous F, Zhang Y, Dipierro C, Kefas B, Johnson E, Marcinkiewicz L, Jiang J, Yang Y, Schmittgen TD, Lopes B, Schiff D, Purow B, Abounader R: MicroRNA-34a inhibits glioblastoma growth by targeting multiple oncogenes. Cancer Res 2009, 69:7569-7576.

36. Wang Z, Banerjee S, Li Y, Rahman KM, Zhang Y, Sarkar FH: Downregulation of notch-1 inhibits invasion by inactivation of nuclear factorkappaB, vascular endothelial growth factor, and matrix metalloproteinase-9 in pancreatic cancer cells. Cancer Res 2006, 66:2778-2784.

37. Dang L, Fan X, Chaudhry A, Wang M, Gaiano N, Eberhart CG: Notch3 signaling initiates choroid plexus tumor formation. Oncogene 2006, 25:487-491.

38. Ellisen LW, Bird J, West DC, Soreng AL, Reynolds TC, Smith SD, Sklar J: TAN1 , the human homolog of the Drosophila notch gene, is broken by chromosomal translocations in T lymphoblastic neoplasms. Cell 1991, 66:649-661.

39. Stylianou S, Clarke RB, Brennan K: Aberrant activation of notch signaling in human breast cancer. Cancer Res 2006, 66:1517-1525.

40. Liu J, Sato C, Cerletti M, Wagers A: Notch signaling in the regulation of stem cell self-renewal and differentiation. Curr Top Dev Biol 2010, 92:367-409.

41. Bin Hafeez B, Adhami VM, Asim M, Siddiqui IA, Bhat KM, Zhong W, Saleem M, Din M, Setaluri V, Mukhtar H: Targeted knockdown of Notch1 
inhibits invasion of human prostate cancer cells concomitant with inhibition of matrix metalloproteinase- 9 and urokinase plasminogen activator. Clin Cancer Res 2009, 15:452-459.

42. Liu Y, Dehni G, Purcell K, Sokolow J, Carcangiu ML, rtavanis-Tsakonas S, Stifani S: Epithelial expression and chromosomal location of human TLE genes: implications for notch signaling and neoplasia. Genomics 1996, 31:58-64.

43. Wang Z, Kong D, Banerjee S, Li Y, Adsay NV, Abbruzzese J, Sarkar FH: Down-regulation of platelet-derived growth factor-D inhibits cell growth and angiogenesis through inactivation of Notch-1 and nuclear factorkappaB signaling. Cancer Res 2007, 67:11377-11385.

doi:10.1186/1476-4598-10-130

Cite this article as: Raghu et al: Specific knockdown of uPA/uPAR

attenuates invasion in glioblastoma cells and xenografts by inhibition of cleavage and trafficking of Notch -1 receptor. Molecular Cancer 2011 10:130.

\section{Submit your next manuscript to BioMed Central} and take full advantage of:

- Convenient online submission

- Thorough peer review

- No space constraints or color figure charges

- Immediate publication on acceptance

- Inclusion in PubMed, CAS, Scopus and Google Scholar

- Research which is freely available for redistribution

Submit your manuscript at www.biomedcentral.com/submit 